\title{
PRISMA
}

\section{A Unique Phonon Spectrometer}

\author{
C. Andreani ${ }^{1}$, U. Steigenburger ${ }^{2}$ and C.G. Windsor ${ }^{3}$ \\ 1 /I Università di Roma, Italy \\ ${ }^{2}$ Rutherford-Appleton Laboratory, Chilton, UK \\ ${ }^{3}$ Harwell Laboratory, Didcot, UK
}

We now have in physics many techniques for revealing the structure of solids down to an atomic scale. However, structure is only the first chapter of the story. We are unlikely to unravel the secrets of high temperature superconductivity from structure alone. We need to be able to "feel" the structure, and sense the forces between the atoms. If we had a ball and stick model of an atomic structure lying on the table before us, what would we do? Many of us might choose an atom, flick it with our forefinger, and watch the structure vibrate. Nature has given us a probe to do exactly this. The momentum change of a neutron scattered by the solid mimics the impulse from our forefinger. Its energy change measures the corresponding frequency of vibration.

The vibrations of a solid are called phonons. Their theory was worked out over 75 years ago by Born, von Karman and Debye, but for 45 years they were familiar only in their bulk form as heat. The race to see them individually almost turned out to be a dead heat. In 1957, groups at Saclay in France, Brookhaven in the USA, and at Chalk River in Canada all saw phonon peaks using neutron scattering. However, it was the Canadian group, led by B.N. Brockhouse, whose method won the day. His famous triple axis spectrometer, whose scattering geometry is illustrated in Fig. 1, used large crystals to define the incoming and outgoing neutron momenta and energies. In a slow but sure way, the phonons could be mapped out, frequency by frequency, momentum by momentum.

Many dispersion curves, giving excitation frequency against momentum, pioneered by Brockhurst have been measured by neutron scattering over the years. The information on the interactions between atoms which these curves give has proved decisive in many branches of solid state physics. They have pointed the way forward to our understanding of structural phase transitions, of magnetism, and even this year to the existence of correlations that may be responsible for high temperature superconductivity.

\section{Pulsed Neutron Sources}

The high flux reactors built during the sixties in the USA and France have yet to be surpassed. Instead, the seventies and eighties have seen the rapid development of pulsed neutron sources. Their obvious advantage over steady state reactors is that the peak neutron production may be very large for a given mean rate of heat removal. Brockhouse's neutron monochromator crystal may be dispensed with, and the incoming neutron energy worked out instead from the time of flight of the

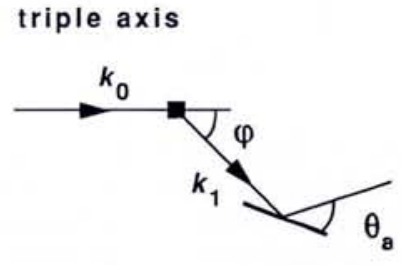

constant $Q$
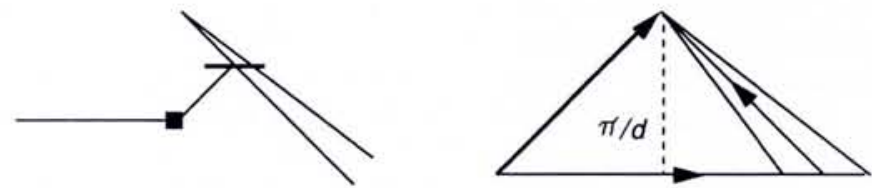

PRISMA, Dubna, MAX
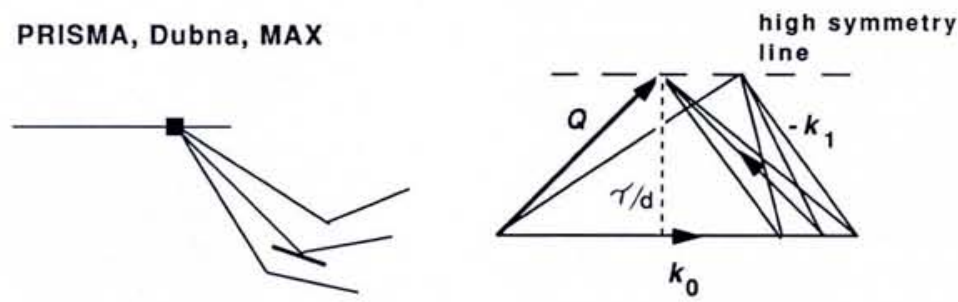

Fig. 1 - The geometries in real space (left) and reciprocal space (right) for the three types of neutron spectrometers that operate at constant momentum transfer: upper - triple axis; middle - constant $Q$; lower - PRISMA. The objective in each case is to obtain a fixed scattering vector $Q=\boldsymbol{k}_{0}-\boldsymbol{k}_{1}$ with a varying energy transfer function $E_{0}-E_{1}=\hbar \omega$ where $\boldsymbol{k}_{0}, E_{o}$ and $\boldsymbol{k}_{1}, E_{1}$ are the wave vectors and energies of the incident and reflected neutrons. The sample scatters at an angle $\varphi$ and the crystal analyser reflects at an angle $2 \theta_{a}$.

For the triple axis spectrometer, used with a reactor source, the constant $Q$ scan is measured sequentially by changing the incident energy at each setting of the sample. For PRISMA, with a pulsed neutron source, constant $Q$ scans can be measured continuously along a line of high symmetry in the sample's reciprocal space. 
a multi-counter instrument able to perform a frequency scan at a given momentum - the "Constant $Q$ Spectrometer" - whose geometry is described in Fig. 1. Independently of the Dubna group they devised a multicounter spectrometer which would give a series of frequency scans at momenta along high symmetry directions. The instrument would be able to map out a whole dispersion curve at one setting. Fig. 2 shows a sketch made by C.G. Windsor in April 1977 of this proposed "high symmetry spectrometer" and its application to the mapping of excitations in chromium (1).

Harwell's source was being rebuilt at this time so the idea of a high symmetry spectrometer was in fact first implemented on the pulsed neutron source at KEK, Tsukuba in Japan. Fig. 3 shows The Multi-Angle crystal spectrometer, MAX, that was installed on the KENS spallation neutron source in 1980 . This instrument has operated well ever since. However, its counters were also rather widely separated so that its frequency scan was relatively sparse.

An Anglo-Italian collaboration began in 1981 with joint experimental work using the Constant $\mathrm{Q}$ Spectrometer on the new Harwell linac. The experiments were difficult - phonons in a single crystal of "heavy" ice were only just observable. When plans for the much more intense ISIS spallation neutron source at the Rutherford-Appleton Laboratory (see Europhys. News 20 (1989) 119) were approved, thoughts of a better instrument began to be bounced around.

Researchers at the Los Alamos spallation source LANCSE were already building an improved constant $\boldsymbol{Q}$ machine. However, at the High Energy Excitations meeting at Los Alamos in 1984, there were many ideas for better instruments. Could one, for example, use an array of small cylindrical analyser crystals or "organ pipes" to achieve the required continuous change of crystal orientation with position? At the International Collaboration of Intense Neutron Sources meeting held in Oxford in 1986, F. Sacchetti unveiled a very flexible constant $\boldsymbol{Q}$ design which could operate in several different modes according to the needs of the experiment. In the end, none of these instruments was built. The physics of high energy (several eV, "hot") neutrons was emerging and the importance of developing an instrument for measuring coherent excitations using the new pulsed sources was strongly felt among the neutron scattering community.

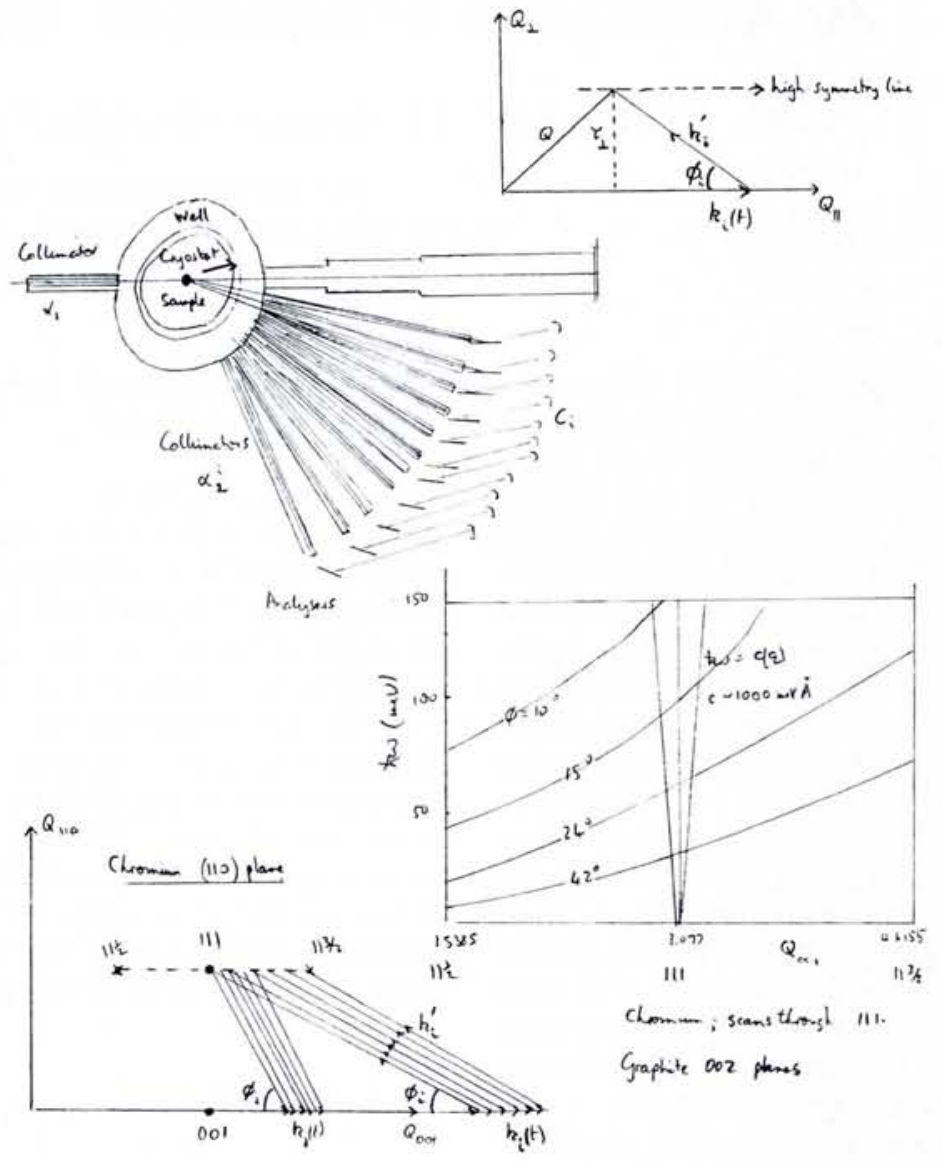

Fig. 2 - Illustrations from a letter written in April 1977 by C.G. Windsor asking for comments on a proposed high symmetry spectrometer shown in the sketch. The upper diagram illustrates the theory where constant $\boldsymbol{Q}$ is maintained along a line of high symmetry. The lower two diagrams show its application in the difficult task of measuring the very steep dispersion line of antiferromagnetic spin waves in chromium.
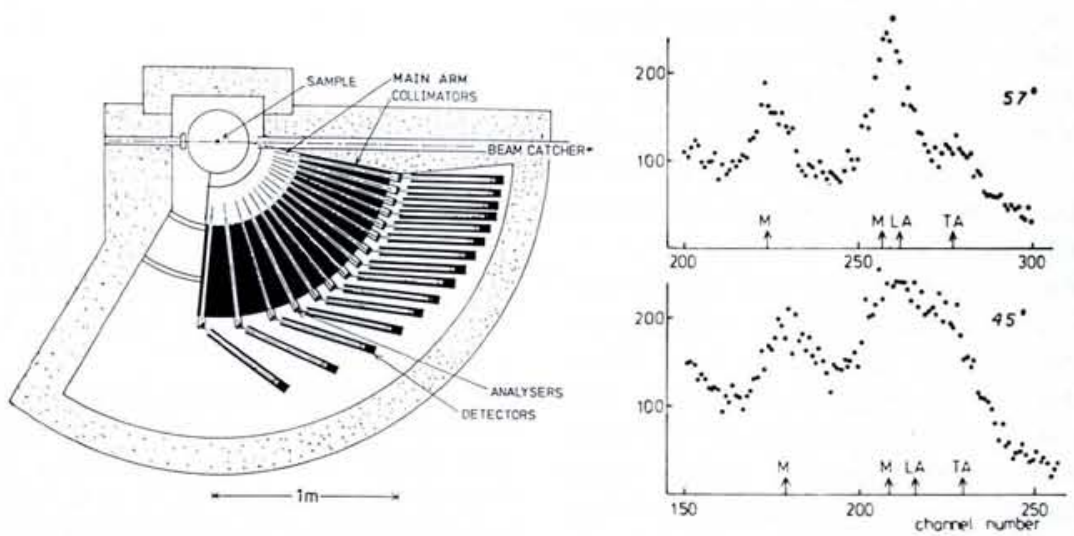

Fig. 3 - The Multi-Angle crystal spectrometer MAX at the KEK pulsed neutron source. To the right are shown time of flight spectra (number of counts versus energy level) for constant $Q$ scans at two scattering angles for iron. The peaks correspond to magnon (M) and longitudinal and transverse accoustic (LA, TA) phonon lines. 


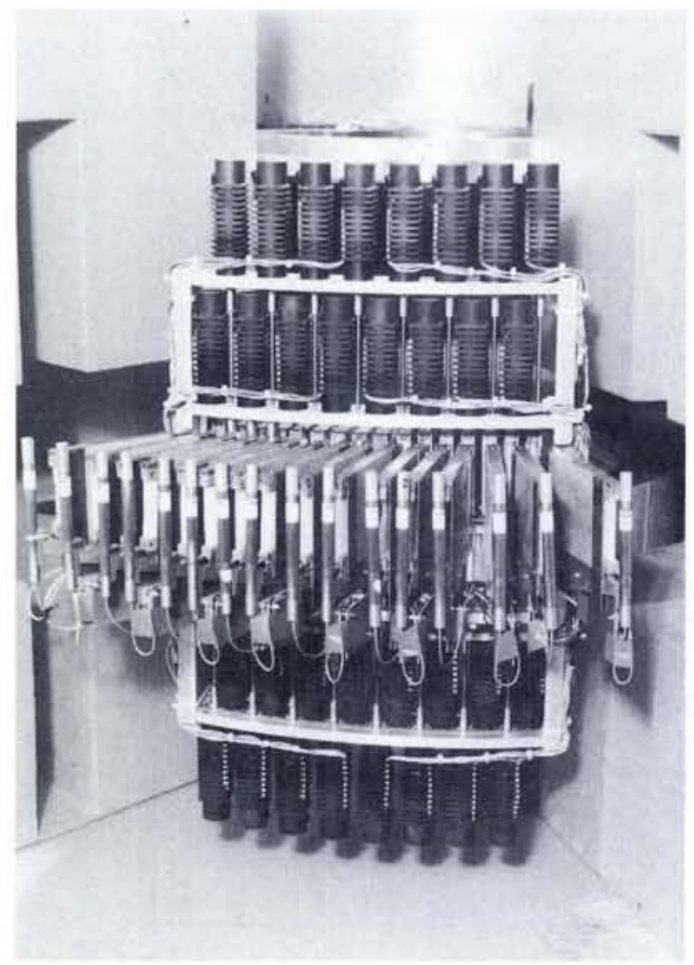

Fig. $4-A$ view from the rear of PRISMA. Motors with their shafts running vertically are arranged in two banks, each with eight motors, at both the top and the bottom of the spectrometer. The upper banks drive the 16 crystal analysers, the lower ones drive the arms of the 16 counters.

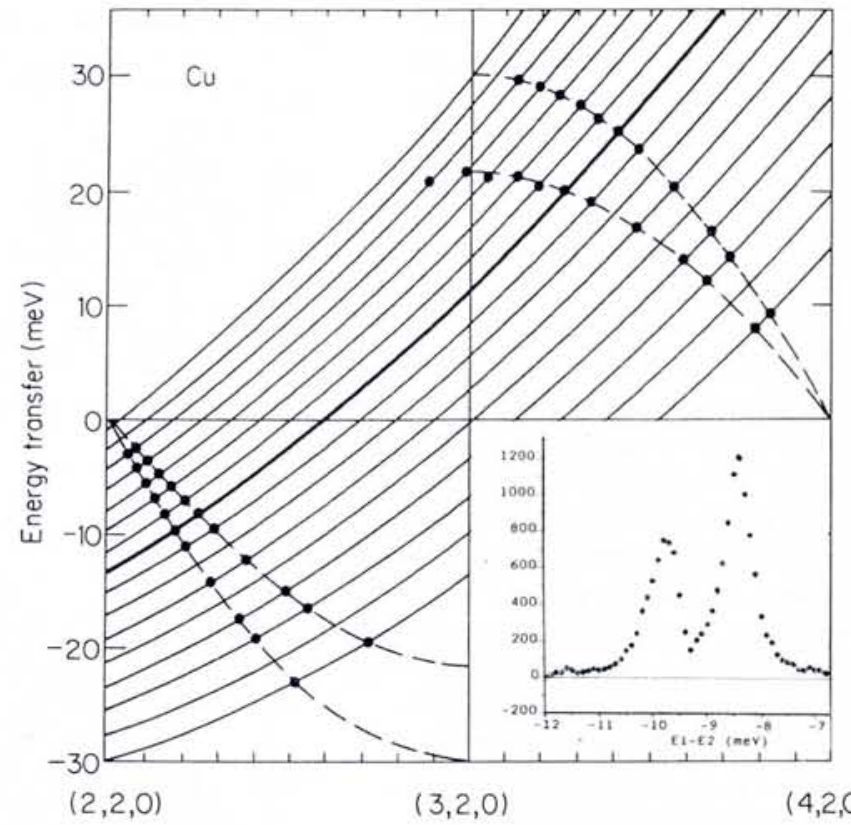

Fig. 5 - Dispersion curves for a single crystal of copper along the [100] direction. The positions of phonon peaks with both negative and positive energy transfers are indicated on lines corresponding to the traces through $Q, \omega$ space for each of the 16 counters of PRISMA. The horizontal axis describes the scattering wave vector $\boldsymbol{Q}$ and is related to the inverse of the amplitude of the excitation. The vertical axis gives the energy transfer $E_{0}-E_{1}=\hbar \omega$ where $\omega$ is the frequency of the excitation. Inset is a portion of the time of flight spectrum for the counter whose locus in $Q, \omega$ space is shown by the heavy line in the main figure. The phonon peaks are marked on the dispersion line.

\section{PRISMA}

PRISMA was designed in the peaceful surroundings of the Institute for the Structure of Matter (ISM) at Frascati on the hills outside Rome (2). It took its name from the PRoject of ISM, and also from its 16 counters which split up the scattered neutron energies just as a glass prism splits up the wavelengths of light. It was one thing to design the instrument, quite another to build it. The design called for the 16 counters to be as close together as possible. The first layouts suggested a mechanical arrangement of unbelievable complexity. The elegant design shown in Fig. 4 was a masterstroke of functional simplicity.

The spectrometer was delivered to the Rutherford-Appleton Laboratory in 1987 , and was soon in place on an ISIS beam line. The problems of an international project emerged. The bits made in different countries did not quite fit. The background of stray neutrons was high. The space behind the counters for shielding off the unwanted neutrons had somehow disappeared during construction. The battle was slowly won. Components were re-machined. The 16 counter arms were cut, and rejoined a centimetre shorter, to allow room for the precious shielding material. The background was steadily reduced as each successive neutron leakage path from source to counter was located and blocked with shielding. It was not until the summer of 1988 that the first phonons were measured on a large single crystal of copper. It was the turn of the British to hold a party and officially inaugurate the instrument. Professors Bernardi and Mitchell, respective Chairmen of the Italian and UK science research councils were there to make the speeches, but the star of the day was PRISMA.

\section{How PRISMA Works}

A crystal analyser instrument on a pulsed source always has a fixed scattered wave vector $\boldsymbol{k}_{1}$ and a variable incident wave vector $\boldsymbol{k}_{0}$ depending on the time of flight. To scan along the chosen direction, the sample must be orientated so that $\boldsymbol{k}_{0}$ lies parallel to the same direction. For the scattering vector $\boldsymbol{Q}=\boldsymbol{k}_{0}-\boldsymbol{k}_{1}$ to lie on the chosen line, the perpendicular distance $\tau_{d}$ must be such that

$\tau_{\mathrm{d}}=\boldsymbol{k}_{1} \sin \varphi=$ a specified constant, where $\varphi$ is the scattering angle between the incident and scattered wave vectors (see Fig. 1).

This might seem a complicated relationship to maintain - but a crystal analyser spectrometer is able to do it with ease. The fundamental equation for the reflection at a scattering angle $2 \theta_{\mathrm{a}}$ of radiation of wavelength $\lambda$ from lattice planes of spacing $d$ by the analyser is the familiar Bragg's law

$$
\lambda=2 d \sin \theta_{\text {a }}
$$

However, $\lambda$ is related to the scattered wave vector through the equation

$$
\lambda=2 \pi / k_{1}
$$

so that Bragg's law may be written $\pi / d=k_{1} \sin \theta_{\mathrm{a}}=$ a constant.

Eliminating $k_{1}$ gives the condition for operation of the spectrometer:

$$
\sin \theta_{\mathrm{a}}=\left(\pi / d \tau_{\mathrm{d}}\right) \sin \varphi .
$$

At any scattering angle $\varphi$ it is possible to choose an analyser angle $\theta_{\mathrm{a}}$ such that the scattering vectors $\boldsymbol{Q}$ lie along the chosen direction. Moreover, the equation does not contain the incident wave vector $\boldsymbol{k}_{0}$ so that all incident energies along the time-of-flight scan have $Q$ values lying along the specified direction in reciprocal space.

Usually there is some choice in the value of the important parameter $\pi / \tau_{d}$. The crystal analyser $d$-spacing may be altered by selecting the most appropriate set of plane spacings. In principle, other types of crystals could be chosen but on PRISMA only germanium crystals with a [110] axis vertical are available. The lines in Fig. 5 show the 
energy transfer $E_{0}-E_{1}$, where $E_{0}$ and $E_{1}$ are the energies of the incident and scattered neutrons respectively. (The energy transfer $E_{0}-E_{1}=\hbar \omega$ where $\omega$ is the frequency of the vibration mode in the crystal.) The variation of the transfer energy with the scattering angle for each of the 16 detectors is plotted in the figure for the [100] direction of copper using the (111) planes of a germanium analyser. The circles indicate where the peaks in the phonon groups were observed by the individual detectors. The two acoustic modes are clearly resolved in this configuration where the neutron energy is both lost and gained. It is interesting to point out that the dispersion can be measured in different Brillouin zones simultaneously, so after correction of the measured intensity for resolution effects and detector efficiency, information on the eigenvalues of the inelastic modes can be extracted.

\section{Applications of PRISMA \\ High energy excitations}

So far we have spoken of experiments that might have been performed on a conventional Brockhouse triple axis spectrometer. However, there are areas where PRISMA provides unique opportunities. For example, the excitations in many materials extend up past thermal energies and require incident neutrons of even higher energies. Although a reactor with a hot source can deliver a respectable flux of neutrons at the required energies, the reflectivity of crystal monochromaters varies as the fourth power of the wavelength. The pulsed neutron source eliminates the need for a monochromator, and allows the neutrons to be used without bringing them into thermal equilibrium at any particular selected temperature.

For instance, the PRISMA spectrometer was specially designed for the demanding task of measuring the excitations in ferromagnetic metals, particularly the classic examples of iron, cobalt and nickel. The magnetic properties of these metals are interesting because they arise from unpaired electron spins which are moving almost freely through the lattice, rather than being bound to a particular atom. The interaction between the spins in these itinerant metals occurs when both electrons happen to lie on the same atom, and so is generally much larger than the interactions between electron spins lying on different atoms. The spin wave dispersion curve is therefore steeper in energy and thus difficult to measure. High energy neutrons are needed; PRISMA comes

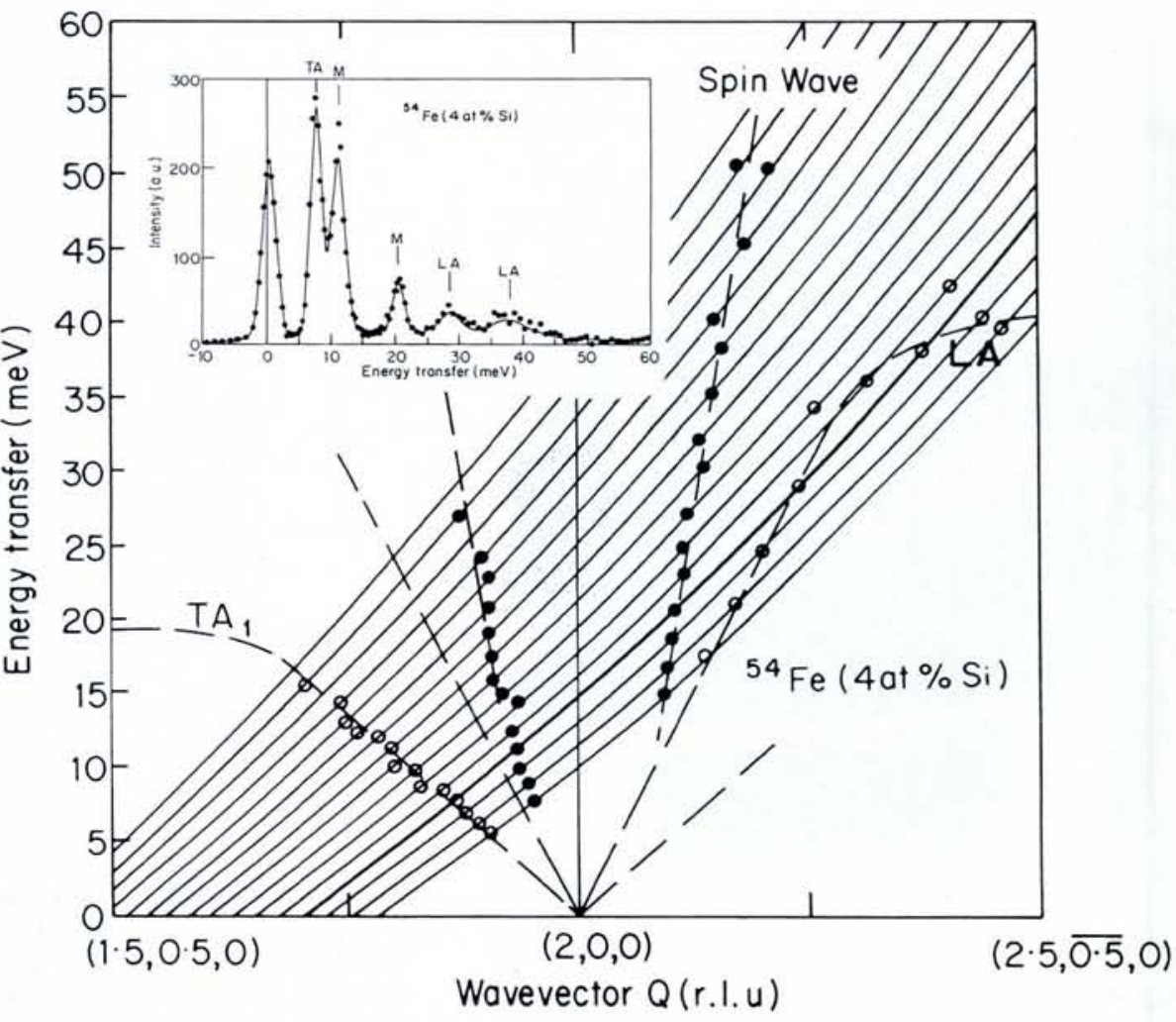

Fig. 6 - Magnon (spin wave) - closed circles - and accoustic phonon (LA, TA) - open circles - dispersion curves in a single crystal of pure ${ }^{54} \mathrm{Fe}$ containing 4 at. \% Si along the [110] direction. Each line corresponds to the trace through the $Q, \omega$ space of one of PRISMA's 16 counters. The time of flight spectrum is for the scan shown by the heavy line given in the inset. Note the very steeply inclined, and difficult to measure, magnon dispersion curve.

to the fore as it does not use a crystal monochromator.

The theory of these excitations was first explored by Stoner in the thirties. $\mathrm{He}$ predicted flat bands of "Stoner modes" at high energies formed by rotating a single electron spin between the up and down bands of the electronic band structure. The theory was later developed by Izuyama, Kim and Kubo, who showed in 1963 that spin waves exist in these materials, but that they should merge into the Stoner modes at high energies.

The energy transfers are high, and because the scattering is magnetic, the scattering vector must be low or the scattering is attenuated by the form factor. Fig. 6 shows both the magnon and accoustic phonon dispersion curves from an iron single crystal. A crystal of ${ }^{54} \mathrm{Fe}$ was used to reduce the multiple phonon scattering. The scan is made along the [110] direction of the reciprocal lattice and the magnons are seen around the (200) reciprocal lattice point. The curve shown required a single day of running on the ISIS source using a $100 \mu \mathrm{A}$ proton current. Our objective will be to follow this dispersion curve upwards to higher energies until the spin waves broaden out and are replaced by a continuous distribution of "Stoner modes".

\section{Temperature dependence}

A second area is formed by experiments where it is important to measure the whole dispersion curve with a single setting of the instrument as, for example, in studying the temperature dependence of dispersion curves. In contrast to the situation for a triple axis spectrometer, neither the sample position nor the instrument settings need be changed during the temperature scan. This is of particular importance when experimenting at very low temperatures where mechanical vibrations can cause significant disturbances to the temperature setting.

Fig. 7 shows the results of measurements of the dispersion curve of the incipient ferroelectric crystal $\mathrm{KTaO}_{3}$. Such a crystal reveals the onset of a structural phase transition by a lowering, or softening, of the phonon energies which correspond to a particular vibration in the crystal. In this case it is the zone centre $\mathrm{TO}_{1}$ mode which corresponds to the atomic motions that characterize the change of structure. As the sample temperature is lowered 


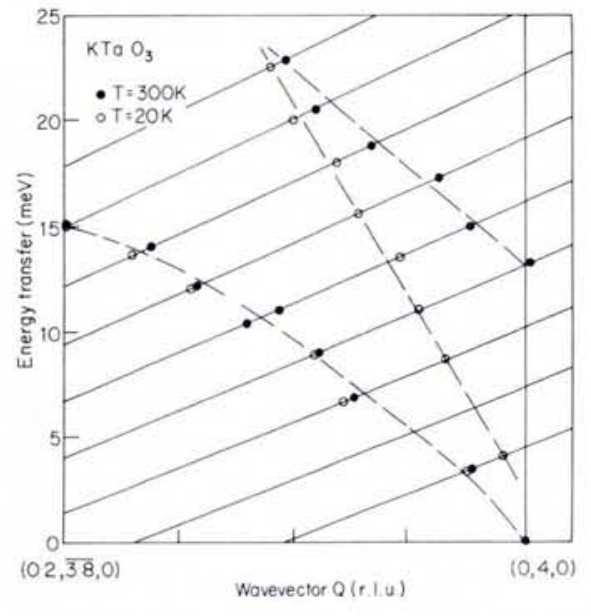

Fig. 7 - The dispersion relation for $\mathrm{KTaO}_{3}$ measured along the [110] direction about the (040) reciprocal lattice point. The full circles correspond to $300 \mathrm{~K}$ and the open circles to $20 \mathrm{~K}$. The observed shift of the dispersion curve of the zone centre TO, mode phonons indicates an increase in the amplitude of the corresponding atomic motions as a phase transition is approached on reducing the temperature.

\section{Edgar Lüscher}

Professor Edgar Lüscher, Chairman of the Metals Section of the EPS Condensed Matter Division, died after a long cruel disease on 16 January 1990.

He was born in Switzerland in 1925 and studied mathematics and physics at the Swiss Federal Institute of Technology in Zürich, and at the University of Lausanne. He received his diploma in 1949, a Ph.D. in physics in 1955 and obtained his habilitation at the University of Lausanne a year later. After teaching and then working for several years in industry, he became a professor at the University of Illinois, Urbana. Since 1964 until his tragic death he was Full Professor of Experimental Physics at the Technical University, München. He was elected in 1965 as member of the Max Planck Society.

His main research fields spanned atomic and condensed matter physics, particularly liquid and amorphous metals. His more recent interests were primarily oriented towards biophysics and chaotic phenomena in general.

We deeply mourn his death, both as a scientist and as a great humanist.

\section{F. Cyrot-Lackmann,}

Grenoble

\section{CORRECTION}

In last month's Directory issue we inadvertently designated E. Jakeman instead of $\mathrm{H}$. Ryde as the Vice-Secretary of the Executive Committee. towards the phase transition, the lower phonon energy means that the amplitude of the corresponding atomic motions increases. As the phonon energy reaches zero at a particular wave vector, the amplitudes of the atomic motions tend to diverge and the phase transition takes place.

\section{Conclusion}

The PRISMA spectrometer has already given encouraging evidence of its potential to measure coherent excitations and the onset of phase transitions at low temperatures. Time will tell if it is eventually able to equal the scientific success of the triple axis spectrometer.

\section{Acknowledgement}

Some of this work formed parts of the scientific programmes of the United Kingdom Atomic Energy Authority, the SERC, and the Italian Consiglio Nazionale delle Richerche.

\section{REFERENCES}

(1) Windsor C.G., Proposal for a High Symmetry Spectrometer, paper submitted to the Spallation Neutron Source Science Planning Group, October 1977.

(2) Andreani C., Carlile C.J., Cilloco F., Petrillo C., Sacchetti F., Stirling G.C. and Windsor C.G., Nucl. Inst. Meth. A254 (1987) 333.

(3) Steigenburger U. et al., Nucl. Inst. Meth. (submitted for publication).

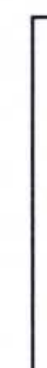

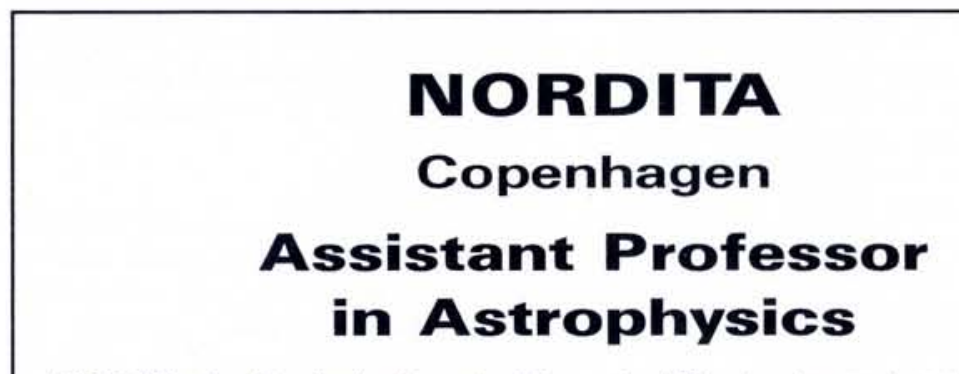

NORDITA, the Nordic Institute for Theoretical Physics, located at the Niels Bohr Institute of Copenhagen University, has an opening for an Assistant Professor in theoretical astrophysics starting in September 1991, or some other date to be agreed upon.

NORDITA is supported by the five Nordic countries, Denmark, Finland, Iceland, Norway and Sweden. Research at the Institute is at present carried out mainly in astrophysics and cosmology, complex systems (including neural nets), condensed matter physics, high energy physics and nuclear physics. There are thus good opportunities to carry out cross-disciplinary studies. Staff members working in astrophysics are K. Enqvist, B. Pagel and C.J. Pethick, whose main interests are in cosmology, the chemical evolution and dynamics of galaxies, high energy astrophysics, and neutron stars. Astrophysicists at NORDITA have close contacts with those at the Niels Bohr Institute, the Danish Space Research Institute, the Copenhagen University Observatory and other institutes in the Nordic area. There are also opportunities for observing at the European Southern Observatory and the Nordic Optical Telescope, among other facilities.

The scientific staff includes six positions as permanent professors, four positions as assistant professors, and Nordic assistant professors. In addition there is a fellowship programme for Nordic graduate students and postdocs. The Institute's activities include an extensive visitor programme for scientists from all over the world, and symposia and summer schools arranged either by NORDITA itself or in cooperation with other Nordic institutes.

The successful applicant is expected to guide fellows at roughly the postdoctoral level, to interact with colleagues at NORDITA and elsewhere in the Nordic countries and to take an active part in the organization of meeting and courses. The position provides excellent opportunities to pursue original research and to have contact with a wide range of developments in theoretical physics. There are good facilities for travelling to other institutes and to meetings, and the Assistant Professor will be encouraged to invite guest scientists to visit NORDITA.

The initial appointment will be for three years, with the possibility of renewal up to a total of six years. The annual salary will be in the range of $240000-310000$ Danish kroner depending on experience.

Those interested in the appointment should send a curriculum vitae, a list of publications and the names of three referees before 1 November 1990 to:

Prof. C.J. Pethick, Director, NORDITA, Blegdamsvej 17, DK-2100 Copenhagen 0. Tel.: ++45-31 4216 16, Fax: ++45-31 3891 57, Email: nordita@nbivax.nbi.dk.

There is no restriction on the nationality of the applicant. Those wishing to recommend suitable candidates are urged to contact the Director. 